\title{
Three-dimensional calibration targets for optical coherence tomography
}

\author{
Michelle Gabriele Sandrian ${ }^{\mathrm{a} *}$, Pete Tomlins ${ }^{\mathrm{b}}$, Peter Woolliams ${ }^{\mathrm{c}}$, Janarthanan Rasakanthan ${ }^{\mathrm{d}}$, Graham \\ CB Lee ${ }^{\mathrm{d}}$, Anna Yang ${ }^{\mathrm{a}}$, Boris Považay ${ }^{\mathrm{a}}$, Aneesh Alex ${ }^{\mathrm{a}}$, Kate Sugden ${ }^{\mathrm{d}}$, Wolfgang Drexler ${ }^{\mathrm{a}}$ \\ ${ }^{a}$ Center for Medical Physics and Biomedical Engineering, Medical University of Vienna, Währinger \\ Gürtel 18-20, 1090 Vienna, Austria \\ ${ }^{\mathrm{b}}$ Clinical and Diagnostic Oral Sciences, Queen Mary University of London, Barts \& The London \\ School of Medicine and Dentistry, London, UK \\ ${ }^{\mathrm{c}}$ Materials Division, National Physical Laboratory, Hampton Road, Teddington, Middlesex, UK \\ ${ }^{\mathrm{d}}$ Photonics Research Group, School of Engineering \& Applied Science, Aston University, Aston \\ Triangle, Birmingham, UK
}

\begin{abstract}
The recent expansion of clinical applications for optical coherence tomography (OCT) is driving the development of approaches for consistent image acquisition. There is a simultaneous need for time-stable, easy-to-use imaging targets for calibration and standardization of OCT devices. We present calibration targets consisting of three-dimensional structures etched into nanoparticle-embedded resin. Spherical iron oxide nanoparticles with a predominant particle diameter of $400 \mathrm{~nm}$ were homogeneously dispersed in a two part polyurethane resin and allowed to harden overnight. These samples were then etched using a precision micromachining femtosecond laser with a center wavelength of 1026 $\mathrm{nm}, 100 \mathrm{kHz}$ repetition rate and $450 \mathrm{fs}$ pulse duration. A series of lines in depth were etched, varying the percentage of inscription energy and speed of the translation stage moving the target with respect to the laser. Samples were imaged with a dual wavelength spectral-domain OCT system $(\lambda=800 \mathrm{~nm}, \Delta \lambda \approx 180 \mathrm{~nm}$, and $\lambda=1325 \mathrm{~nm}, \Delta \lambda \approx 100 \mathrm{~nm})$ and pointspread function of nanoparticles within the target was measured.
\end{abstract}

Keywords: Calibration target, optical coherence tomography, femtosecond inscription, point-spread function

\section{INTRODUCTION}

The rapidly increasing number of optical coherence tomography (OCT) applications in medical imaging has led to a subsequent need for a simple, reliable and rapid approach that can be universally applied to assess system performance potentially allowing for comparison across systems. There have been multiple attempts to produce tissue-mimicking phantoms ${ }^{1}$, but this remains a challenge given the complexity of tissue, differences in optical properties within a single organ or across subjects, and changes in optical properties due to disease progression.

Recently, multiple groups have focused on the design and fabrication of widely applicable solid, unchanging, targets for three-dimensional OCT image calibration. These can be divided into two general categories: randomly dispersed subresolution point scatterers ${ }^{2,3}$ and well-defined microstructures ${ }^{4,5}$. Many of these studies have focused on the use of these targets for the measurement of point-spread function (PSF), as images represent a convolution of the PSF and the true object. Woolliams et al produced a target using a two-part polyurethane resin doped with iron oxide nanoparticles ${ }^{2}$. They measured PSF and then used deconvolution techniques to correct and improve axial and lateral resolution. Agrawal et al produced a nanoparticle-embedded target consisting of $\sim 200 \mathrm{~nm}$-diameter spherical gold nanoshells in silicone, and used this to measure 3D PSF and variations in PSF with source bandwidth.

While these randomly dispersed sub-resolution point scattering targets can be used to measure 3D PSF, the

*michelle.sandrian@meduniwien.ac.at; phone: +43 140400 1715; fax: +43 1404003988

Optical Diagnostics and Sensing XII: Toward Point-of-Care Diagnostics; and Design and Performance Validation of Phantoms Used in Conjunction with Optical Measurement of Tissue IV, edited by Robert J. Nordstrom, Gerard L. Coté, Proc. of SPIE Vol. 8229, 822914 - (c) 2012 SPIE · CCC code: 1605-7422/12/\$18 · doi: 10.1117/12.907748 
backscattering cross-section of nano- or microparticles can be low and there is a possibility of clustering of particles during mixing, which would effectively result in large variations of particle size. The use of lithographic castings ${ }^{4}$ or inscribed lines ${ }^{5}$ to produce microscopic landmarks has been demonstrated. These techniques allow for precise control over target object location and consistency from target to target. The lithographic casting method, however, has to this point only been shown to produce relatively large features $(\sim 100-200 \mu \mathrm{m})$ and feature spacing $(\sim 45 \mu \mathrm{m})$, which prohibits its use in PSF analysis. Femtosecond inscription techniques allow for more precisely defined features, with laser markings on the order of 3-4 $\mu \mathrm{m}$ having been demonstrated.

In this paper, we present the design of an imaging target that combines both categories of solid, three-dimensional OCT targets: a nanoparticle-embedded resin calibration target etched using femtosecond laser techniques.

\section{MATERIALS AND METHODS}

\subsection{Target fabrication}

One milligram of spherical red iron oxide nanoparticles ranging in size from 300-800 nm (predominant diameter of 400nm; Polysciences, Inc.,Warrington, PA, USA) was added to 120g polyurethane resin PU3660SB (Alchemie Ltd, Warwick, England). The mixture was sonicated for 15 minutes at room temperature, followed by the addition of $100 \mathrm{~g}$ PU3660SA. This was poured into molds, placed in a vacuum for approximately 15 minutes to remove excess air, allowed to harden for one hour at room temperature and then placed at $50^{\circ} \mathrm{C}$ overnight.

After hardening, one sample (mold size: $65 \times 35 \mathrm{~mm}$ ) was inscribed using a femtosecond micromachining laser (Oxford Lasers Ltd, UK) with a center wavelength of $1026 \mathrm{~nm}, 100 \mathrm{kHz}$ repetition rate and sub-450 fs pulse duration. The maximum laser power was attenuated $83 \mathrm{~mW}$ to accommodate etching of polyurethane. An inscription lens with a numerical aperture of 0.5 and focal length of $2.0 \mathrm{~mm}$ was used (Mitutoyo M Plan Apo NIR 100X) for a theoretical spot size of $\sim 2.5 \mu \mathrm{m}$. A series of lines covering an energy range of 5-100\% of total energy (evenly sampled step size of $6.8 \%$, $\sim 42-830 \mathrm{~nJ}$ ), were etched, with each energy level repeated twice (see schematic in Figure 1). At each inscription point, 12 lines in depth were inscribed at for energy level. Lines were offset by $75 \mu \mathrm{m}$ in depth (axially) and $25 \mu \mathrm{m}$ laterally using a computer-controlled multi-axis translation stage. A photograph of the final inscribed and nanoparticle-embedded target can be seen in Figure 2.

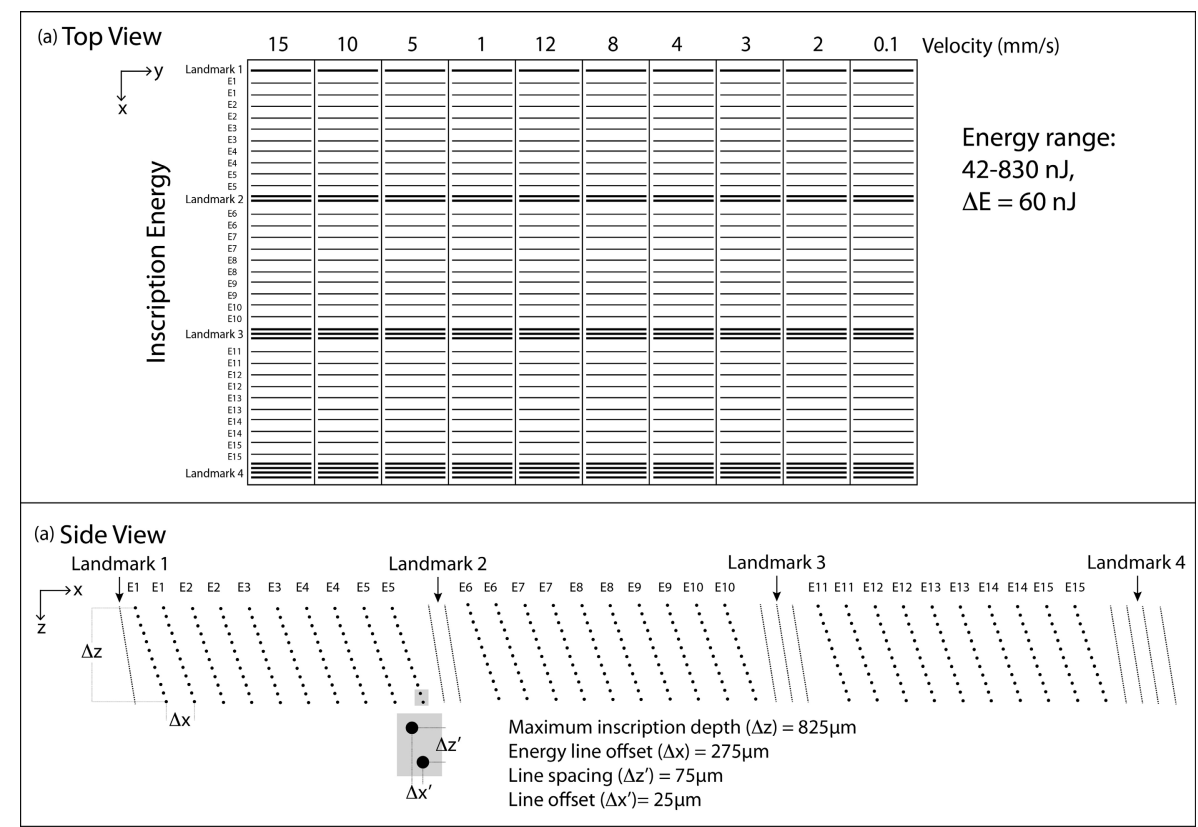

Figure 1. Design of femtosecond laser inscription points for 3D OCT calibration target (a) top view, showing range of velocity (translation stage speed) and inscription energy, and (b) side view, showing variation in depth. 


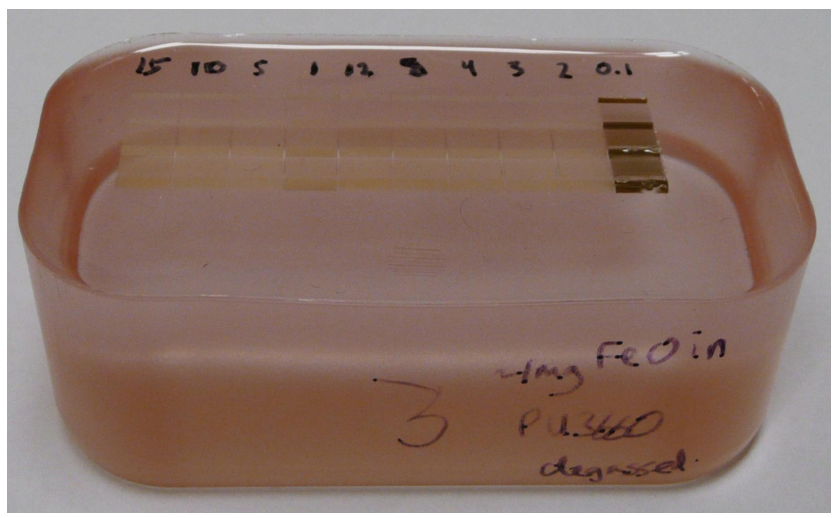

Figure 2. Photograph of iron oxide nanoparticle-embedded femtosecond inscribed calibration target. Each column corresponds to a different inscription speed, as indicated by the handwritten labels. At a translation stage speed of $0.1 \mathrm{~mm} / \mathrm{s}$, damage to the target is clearly evident.

\subsection{Microscopic analysis}

An optical microscope (Axioscope 2, Carl Zeiss, Germany) with a 40X magnification lens was used to photograph and measure the inscription lines in the target. The line widths corresponding to different energy levels and depths were recorded. Figure 3 shows examples of various defects in the polyurethane nanoparticle embedded target. At a translation stage speed of $15 \mathrm{~mm} / \mathrm{s}$ and low energy $(42 \mathrm{~nJ}), 8$ lines were observed as opposed to 12 that were expected. This can be explained by the combination of low energy, high speed and depth, which results in a reduced incident intensity exposure. With increased depth, the lines change from dense and continuous to transparent, and finally, when below the material threshold for damage, no visible line. On the contrary, when using a combination of higher energy (437 nJ) and keeping the translation speed constant, lines are dense and irregular.

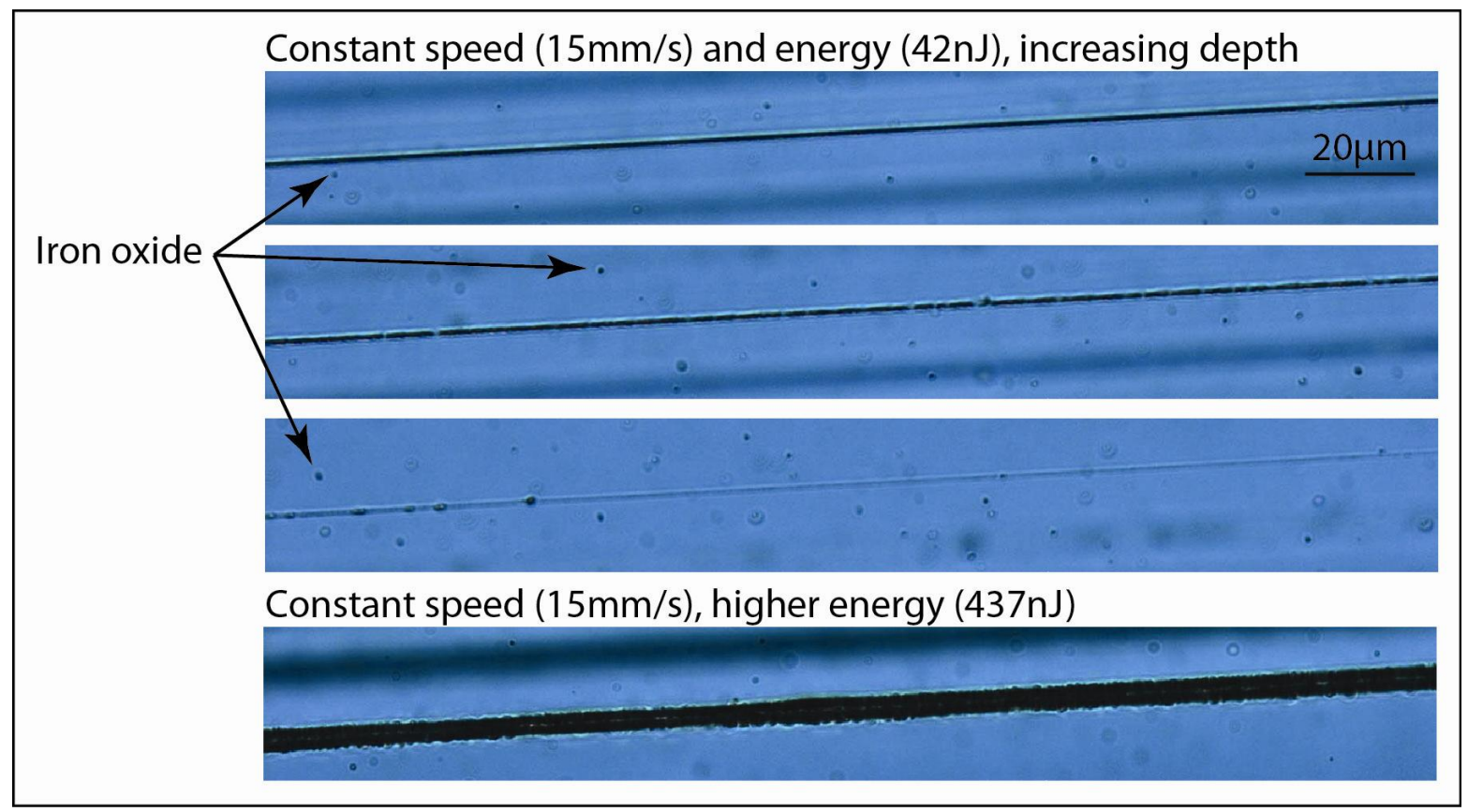

Figure 3. Microscopic image of inscription lines demonstrating differences that result from changing inscription energy and depth. 
Figure 4 shows the measured width of multiple lines and energy levels at two different translation stage speeds, $15 \mathrm{~mm} / \mathrm{s}$ and $10 \mathrm{~mm} / \mathrm{s}$. This figure shows that at a constant speed, line width increases with energy, as expected. At a speed of 15 $\mathrm{mm} / \mathrm{s}$ and the lowest energy level $(41.5 \mathrm{~nJ})$, only 8 lines were distinguishable by microscopic evaluation. This figure demonstrates that, to obtain sub-resolution $(\sim 3 \mu \mathrm{m})$ line size, a combination of fast translation stage speed (eg, $15 \mathrm{~mm} / \mathrm{s}$ ) and an energy range of $\sim 50-100 \mathrm{~nJ}$ is necessary. In addition, at a given energy level, differences in line width can be observed that are related to focusing of the inscription lens in the target.

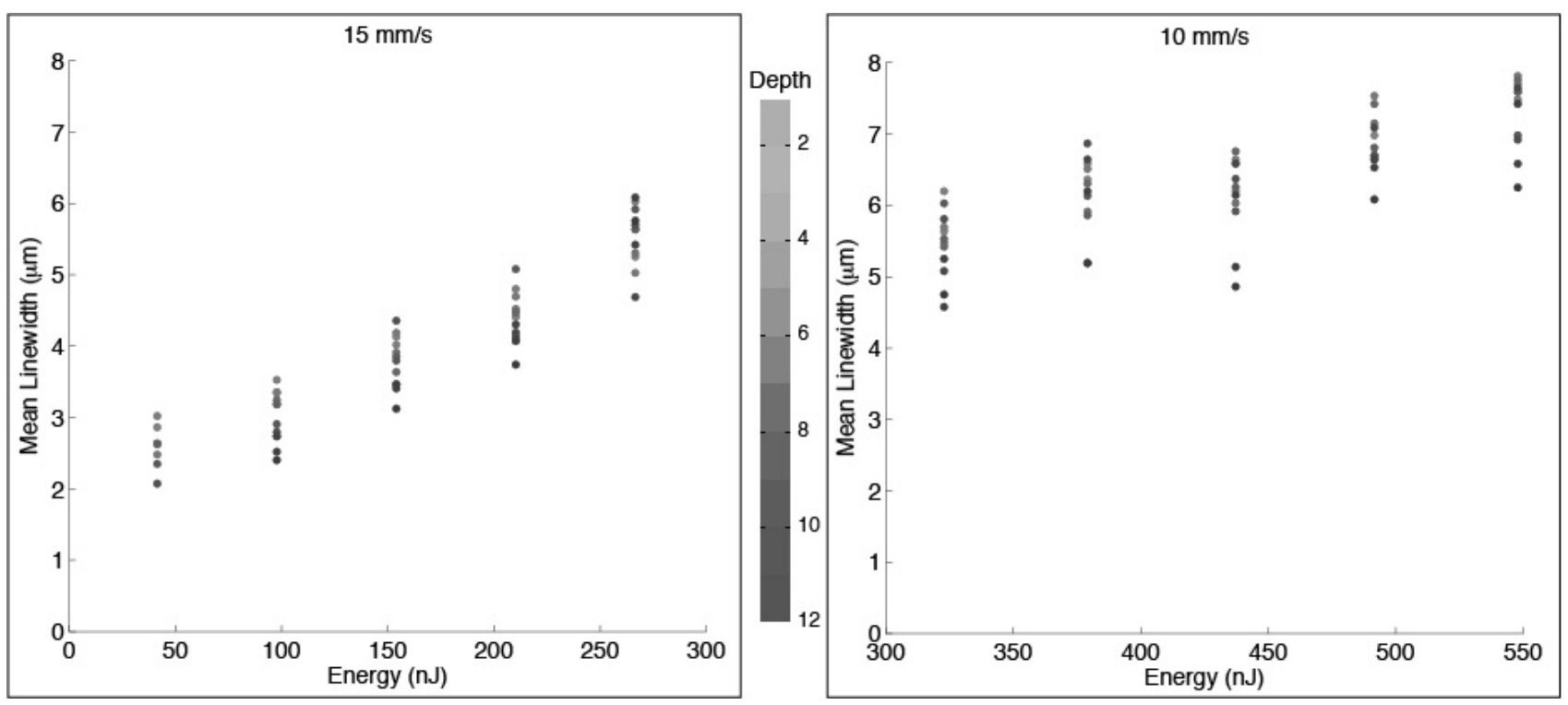

Figure 4. Mean line width versus inscription energy for translational stage speeds of $15 \mathrm{~mm} / \mathrm{s}$ (left) and $10 \mathrm{~mm} / \mathrm{s}$ (right). Each energy level consists of up to 12 measurements, corresponding to 12 possible depth measurements and indicated by darker shading. Individual points represent single lines, and darker-shaded points are located deeper in the target.

\subsection{OCT imaging and analysis}

The calibration target was imaged using a simultaneous dual wavelength OCT system (800 and $1320 \mathrm{~nm})$, which has previously been described in detail. ${ }^{6}$ This system consists of two spectrometer-based frequency-domain OCT systems and uses an ultra-broadbandwidth Ti:Sapphire laser with a center wavelength of $800 \mathrm{~nm}$ and bandwidth of $150 \mathrm{~nm}$ (Femtolasers $\mathrm{GmbH}$, Vienna, Austria) and a superluminescent laser diode source with a center wavelength of $1320 \mathrm{~nm}$ and bandwidth of $100 \mathrm{~nm}$ (Thorlabs, Inc, Newton, NJ, USA). Both systems have an A-scan acquisition rate of $47 \mathrm{kHz}$. The theoretical axial resolutions of the $800 \mathrm{~nm}$ and $1320 \mathrm{~nm}$ systems are $\sim 2 \mu \mathrm{m}$ and $8 \mu \mathrm{m}$, respectively, and the theoretical transverse resolutions are 24 and $20 \mu \mathrm{m}$, respectively. The dual wavelength system has been designed for clinical use, so the two beams scan approximately the same volume. High-density images were acquired (1000x500x 1024 pixels), covering an area of approximately $4.9 \times 2.5 \times 2.3 \mathrm{~mm}$ to give a lateral A-scan spacing of $\sim 5 \mu \mathrm{m}$. After acquisition, spectral measurements were inverse Fourier-transformed and processed to obtain an intensity-based OCT image.

Images were then processed to obtain multidimensional PSF measurements. To do this, background noise was first subtracted using mean background intensity information and top hat filtering. Morphological operations were used to fill in small gaps between neighboring structures, and objects (laser etched marks and nanoparticles) were located in 3D using blob detection. Blob properties including volume and location in 3D space were measured, and structures were sorted by volume such that larger volume structures, such as etched lines and landmarks, could be distinguished from smaller-volume structures (i.e., nanoparticles).

A multivariate normal distribution was fitted to each nanoparticle, using the centroid and volume determine by blob detection and localized volumetric intensity information from corresponding location in the unfiltered image. This provided a mean (centroid of each nanoparticle) and corresponding variance, which was used to calculated full-width at 
half maximum (FWHM) PSF in $\mathrm{x}, \mathrm{y}, \mathrm{z}$. A volumetric region of interest was defined on the central slice of the image stack from both the $800 \mathrm{~nm}$ and $1300 \mathrm{~nm}$ images. This region the surface and the first etched line, was manually selected for PSF measurements, as it contained only nanoparticles.

\section{RESULTS}

Cross-sectional OCT images from both wavelengths can be seen in Figure 5. Landmark 1 (densely inscribed line in depth on the right side of Figure 5) and landmark 2 (two dense lines on the left) indicate the scan location corresponds to energy levels 1-5 (42-267 $\mathrm{nJ}$ ), with ten columns of inscribed lines ( $2 \mathrm{x}$ each energy level). The deeper penetration of the longer wavelength $(1320 \mathrm{~nm})$ is evident when comparing the two images, with all twelve etched lines visible for some energy levels, while only 4 lines in depth could be clearly discerned in the $800 \mathrm{~nm}$ image. From the image, it is clear that two beams were misaligned, with two corresponding cross-sections allowing the lateral beam offset to be measured to be $0.45 \mathrm{~mm}$. The difference in contrast of structures (nanoparticles and etched marks) between systems, which is evident in Figure 5, may be explained by the backscattering cross-sectional profile of iron oxide nanoparticles as well as localized changes in index of refraction caused by inscription in polyurethane that may have been more easily visualized in the $1320 \mathrm{~nm}$ system.

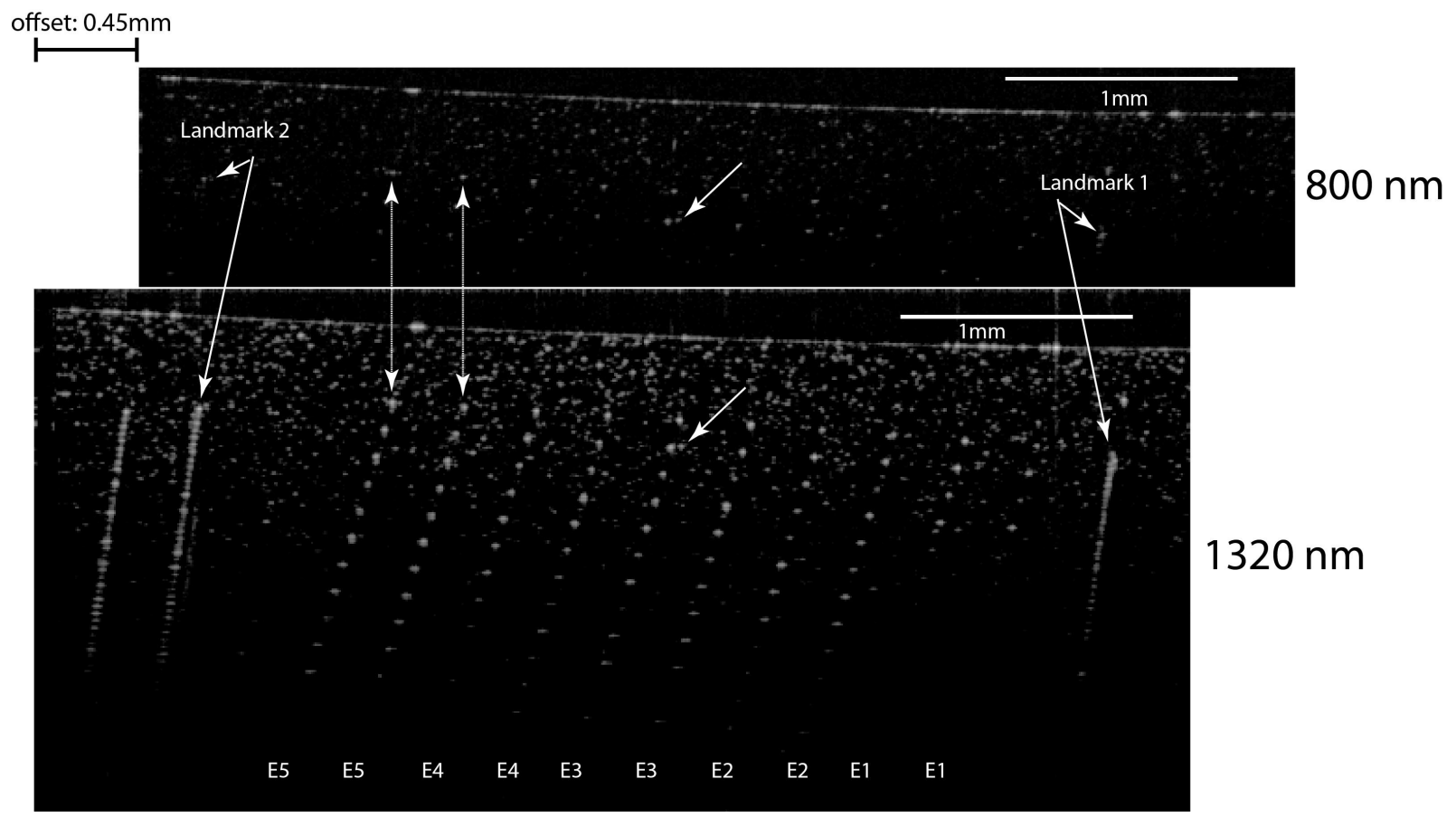

Figure 5. OCT images acquired simultaneously using dual wavelength system; corresponding features are highlighted (arrows) and energy levels 1-5 are labeled (E1-E5). An offset between images of $0.45 \mathrm{~mm}$ was measured, and the increased penetration depth for the $1320 \mathrm{~nm}$ system is evident by the number of etched landmarks visible in depth.

Histograms from three-dimensional PSF measurements are shown in Figure 6. The lateral measurements were expected to be similar since the scans were isometrically sampled in $\mathrm{x}$ and $\mathrm{y}$, and this is evident for both wavelengths. The most frequent (highest count) axial resolution from the $800 \mathrm{~nm}$ images was $3.0 \mu \mathrm{m}$, and $29.8 \mu \mathrm{m}$ for both $\mathrm{x}$ and y lateral resolution. In the $1320 \mathrm{~nm}$ image, the most frequent axial resolution was $13.04 \mu \mathrm{m}$, while the lateral resolution was 28.7 $\mu \mathrm{m}$ in $\mathrm{x}$ and $29.4 \mu \mathrm{m}$ in $\mathrm{y}$. Axial $1320 \mathrm{~nm}$ system PSF measurements and lateral resolution measurements from both wavelengths were substantially higher than the theoretical expected values; this indicates that the system adjustments may need to be made. In addition, fine-tuning the algorithm and adjusting scaling factors based on standard calibration techniques are necessary to compare our results to expected values. 


\section{$800 \mathrm{~nm}$}
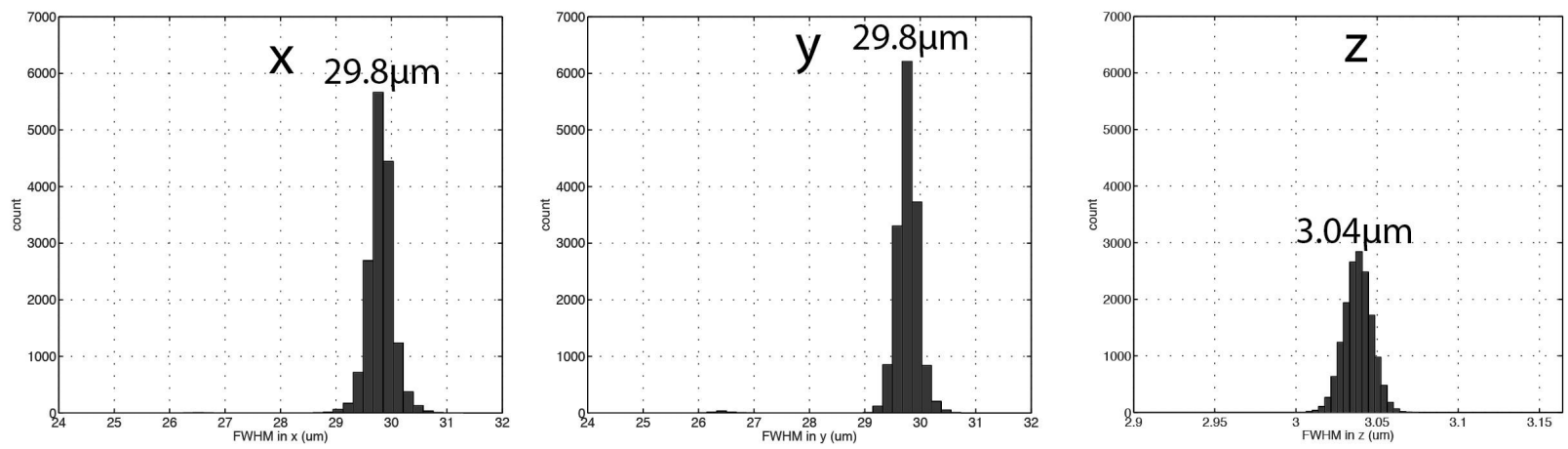

$1320 \mathrm{~nm}$
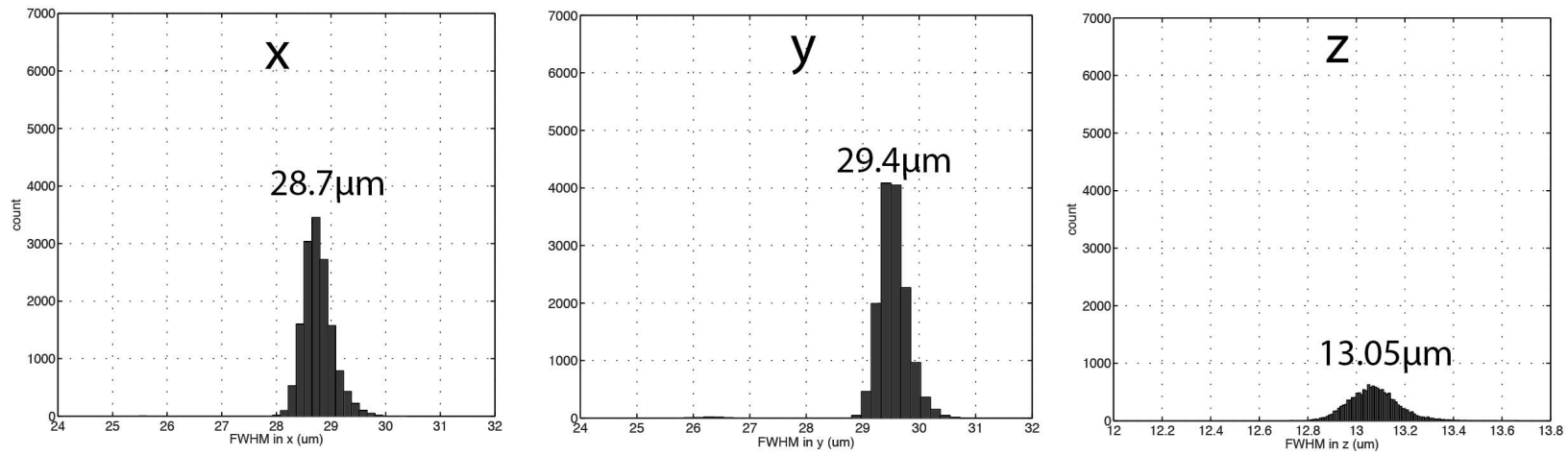

Figure 6. Histogram showing the number of nanoparticles with a given FWHM measurement after Gaussian fitting in $3 \mathrm{D}$.

\section{CONCLUSION}

We developed a nanoparticle-embedded, femtosecond inscribed 3D imaging calibration target and showed preliminary steps toward implementing it in the evaluation of OCT system performance using an automated assessment of PSF in 3D from multiple structures within the target with respect to known landmarks. A fully automated automated assessment of the entire image, including etched marking size and location, could further improve the utility of this calibration target.

While randomly dispersed nanoparticle targets offer the advantage of sub-resolution points from which to estimate PSF, there are some disadvantages to this approach, including the possibility of aggregation of particles, which may result in a larger-than-resolution particle depending on the degree of clustering. From our images, it is clear that there is a distribution of particle size, which indicates there is some aggregation or distribution of particle size. However, an advantage of this combine target with known locations of inscribed micron-sized etching, it that is possible to return to an exact volume within the target to characterize the number and size of iron oxide nanoparticles. This was not possible in previous nanoparticle-embedded targets.

In this work, we demonstrated the use of femtosecond-inscribed lines as landmarks and reference points, but if line width can be designed to be consistent in depth using a combination of focusing adjustment, energy and speed, it might be possible to use inscription lines for PSF measurements in depth, as an indication of system sensitivity, and for measurements of signal roll-off with depth. However, given the requirement for a high NA inscription lens to achieve micron-scale defects, the achievable inscription depth is limited, so even if the line width in depth remains consistent, the addition of nanoparticles could still be use for assessing additional parameters such as resolution decay.

In summary, our novel design offers a relatively straightforward approach to assessing OCT systems in 3D. While this will initially be useful for evaluating system status over multiple measurement time points, it also has potential for 
standardization across devices. It should be possible to use this target to evaluate additional parameters such as field of view, depth roll-off, distortion, linearity, resolution decay, and sensitivity.

\section{REFERENCES}

[1] Pogue BW and Patterson MS, "Review of tissue simulating phantoms for optical spectroscopy, imaging and dosimetry," J. Biomed. Opt. 11(4), 041102 (2006).

[2] Woolliams PD, Ferguson RA, Hart A, Grimwood A and Tomlins PH, "Spatially deconvolved optical coherence tomography,” Appl. Opt. 49(11), 2014-2021 (2010).

[3] Agrawal A, Pfefer TJ, Gilani N and Drezek R, "Three-dimensional characterization of optical coherence tomography point spread functions with a nanoparticle-embedded phantom,” Opt. Lett. 35(13), 2269-2271 (2010).

[4] Curatolo A, Kennedy BF, Sampson DD, "Structured three-dimensional optical phantom for optical coherence tomography," Opt. Exp., 19(20), 19480-19485 (2011).

[5] Tomlins PH, Smith GN, Woolliams PD, Rasakanthan J and Sugden K, "Femtosecond laser micro-inscription of optical coherence tomography resolution test artifacts," Biomed. Opt. Exp. 2(5), 1319-1327 (2011).

[6] Alex A, Weingast J, Hofer B, Eibl M, Binder M, Pehamberger H, Drexler W and Považay B, "3D optical coherence tomography for clinical diagnosis of nonmelanoma skin cancers," Imaging in Medicine, 3(6), 653-674 (2011). 